Original Paper http://ajol.info/index.php/ijbcs http://indexmedicus.afro.who.int

\title{
Transformation et commercialisation des chips de banane plantain au Cameroun : une activité artisanale à forte valeur ajoutée
}

\author{
Guillaume Hensel FONGANG FOUEPE ${ }^{1 *}$, Denis Pompidou FOLEFACK ${ }^{2}$, \\ Zobel PANE PAGUI ${ }^{1}$, Achille BIKOI ${ }^{2}$ et Pascal NOUPADJA ${ }^{2}$ \\ ${ }^{1}$ Faculté d'Agronomie et des Sciences Agricoles, Université de Dschang, B.P 222 Dschang, Cameroun. \\ ${ }^{2}$ Centre Africain de Recherches sur Bananiers et Plantains (CARBAP) B.P. 832 Douala, Cameroun. \\ *Auteur correspondant, E-mail: guillaumefongang@yahoo.fr
}

\section{REMERCIEMENTS}

Nous remercions grandement, le projet IRAD C2D/PAR Plantain qui a financé ce travail.

\section{RESUME}

Les chips de banane plantain jouent un rôle important dans la constitution des revenus des ménages modestes dans les régions du Centre et du Littoral au Cameroun. Au regard de l'émergence de cette filière, une analyse du système de transformation et de commercialisation des chips de banane plantain a été réalisée. Les données ont été collectées à travers une enquête diagnostique par questionnaire auprès de 327 acteurs. Les résultats montrent que la production et la commercialisation des chips de banane plantain sont essentiellement féminines. Les producteurs et commerçants sont relativement jeunes. La production des chips de banane plantain reste encore artisanale et familiale avec un prix moyen de vente de 100 FCFA le sachet. La demande est forte aux points de péage routier $(80 \%)$, dans les agences de voyage $(9 \%)$ et devant les bureaux administratifs (5\%). Bien que l'activité s'avère stimulante, elle est soumise à plusieurs contraintes. Assurer une formation des producteurs et commerçants de chips sur les techniques modernes de transformation, de conditionnement et de commercialisation contribuerait à améliorer les revenus des acteurs.

(C) 2016 International Formulae Group. All rights reserved.

Mots clés: Transformation, commercialisation, chips, banane plantain, Cameroun.

\section{Processing and marketing of plantain chips in Cameroon: A high value-added artisanal activity}

\section{ABSTRACT}

Plantain chips play an important role in income generation of poor households in the Centre and Littoral regions of Cameroon. Given the emergence of this sector, an analysis of the plantain chips processing and marketing system was performed. Data were collected using a diagnostic survey by questionnaires 
addressed to 327 stakeholders. The results show that plantain chips production and marketing is essentially done by women. Producers and traders are relatively young. Plantain chips production remains artisanal and domestic with an average selling price of 100 CFA F per sachet. The demand is high at road toll points (80\%), in travelling agencies (9\%), and in front of administrative offices $(5 \%)$. Though the activity proves to be attractive, it is subjected to several constraints that slow down the sector development. Ensuring chips producers' and traders' training in modern processing, packaging and marketing techniques would contribute to improving stakeholders' incomes.

(c) 2016 International Formulae Group. All rights reserved.

Keywords: Processing, marketing, chips, plantain, Cameroon.

\section{INTRODUCTION}

La banane plantain est une culture vivrière importante d'Afrique Centrale et Occidentale. Elle occupe une place importante dans l'alimentation des populations de plusieurs pays de la Communauté Economique et Monétaire de l'Afrique Centrale (CEMAC) tels que le Cameroun, le Congo, le Gabon, la Guinée Equatoriale et la République Centrafricaine (Nkendah et al., 2007 ; Nkendah et al., 2011 ; Houngbo, 2014 ; Wandji, 2015).

$\mathrm{Au}$ Cameroun, la production de la banane plantain occupe le $8^{\text {ème }}$ rang des productions vivrières (FAO, 2010). La filière banane plantain tient son importance au nombre d'acteurs impliqués (plus de 600000 producteurs et environ 40.000 commerçants), à la production annuelle obtenue $(1,4$ millions de tonnes) et à la contribution à l'économie nationale de l'ordre de 2\% du Produit Intérieur Brut (Efandem et al., 2005).

La demande de la banane plantain est en pleine croissance au Cameroun, en relation avec la pression démographique, l'accroissement de la population urbaine et à cause de ses diverses formes de consommation (Nkendah et Temple, 2003; Nkendah et al., 2007). La transformation de la banane plantain est créatrice de valeur ajoutée importante pour les acteurs. Les différents modes de transformation et d'utilisation du plantain inventoriés sont: les chips de plantain, les bananes séchées, les beignets et gâteaux à base de farine de plantain, le pain, le sel gemme à base de peau de banane plantain, les tourteaux de banane plantain (pour l'alimentation du bétail), les granules de banane plantain, les pattes alimentaires à base de farine de plantain, les crêpes à base de farine de plantain (Planta Innovation, 2011 ; Bikoï et al., 2012).

Le produit de la transformation de la banane plantain le plus rencontré sur les marchés camerounais reste les chips. Elles présentent plusieurs intérêts : une technologie facile et peu coûteuse, un produit de qualité facile et un marché en pleine expansion (Tchango et al., 2009). Ces chips sont de plus en plus consommées au Cameroun comme on peut le constater à travers les points de vente (gares routières, péages routiers, boutiques...). Cette transformation en chips entraîne avec elle l'émergence d'une diversité d'acteurs en particulier les producteurs de chips et les revendeurs.

Malgré cette importance socioéconomique, peu de recherches ont été conduites sur la transformation et la mise en marché des chips de banane plantain. Des informations pertinentes sur cette filière sont très rares voir indisponibles. Or, l'actualisation de la base de données sur les produits transformés de banane plantain permettrait de produire de l'information susceptible d'intéresser divers opérateurs économiques désirant investir dans cette filière. D'autre part, ces informations permettraient de mieux gérer les rétroactions des défaillances du marché sur la production et la transformation du plantain, ce qui contribuerait à une meilleure valorisation de la productivité du travail au niveau de chaque maillon de la chaîne de production- 
transformation-commercialisation avec tous ses intermédiaires (Folefack et al., 2008). Ces informations sont aussi capitales pour toute politique ou stratégie de développement de la filière banane plantain.

La présente étude a pour objectif de faire un état des lieux des systèmes de production et de commercialisation des chips de banane plantain dans les régions du Centre et du Littoral au Cameroun en vue de fournir aux décideurs, aux organismes de recherche et aux acteurs de développement des informations fiables et actualisées susceptibles d'orienter les actions de recherche, de développement et de lutte contre la pauvreté.

\section{MATERIEL ET METHODES \\ Echantillonnage et collecte de données}

Cette étude a été réalisée dans les régions du Centre et du Littoral au Cameroun, notamment dans les villes de Yaoundé, Douala et les postes de péage de Boumnyebel et d'Edéa. Ces villes ont été choisies du fait de l'importance de l'activité de transformation et commercialisation des chips. Les deux points de péage routier ont été choisis à cause de l'importante activité de commercialisation des chips de plantains qu'ils enregistrent.

Les données de cette étude ont été collectées au moyen d'enquêtes par questionnaire entre mars et juillet 2015. L'enquête par questionnaire a concerné près de 327 acteurs, soit 73 producteurs et 254 commerçants des chips de banane plantain dans les villes de Douala, Yaoundé, et au niveau des postes de péages de Boumnyebel et Edéa. Les données collectées portaient sur les caractéristiques socio-économiques des acteurs, les techniques de transformation de la banane plantain en chips, les équipements, les matières premières, les coûts de production, les sources d'approvisionnement, les lieux de vente, les marges bénéficiaires, les contraintes, etc.

L'entretien auprès des chercheurs du CARBAP a concerné les variétés de banane plantain recommandées pour la production des chips, l'équipement moderne pour la production, etc. En outre, les observations directes et la littérature disponible ont aussi été des sources importantes de données.

\section{Analyse des données}

Les données obtenues au moyen des guides d'entretien ont fait l'objet d'analyse thématique. Pour les données individuelles collectées par questionnaire, les statistiques descriptives (fréquence, moyenne, écart type) ont été principalement utilisées. Le compte d'exploitation a été utilisé pour analyser la rentabilité de la transformation et de la commercialisation des chips de banane plantain.

\section{RESULTATS}

\section{Caractéristiques socio-économiques des acteurs}

Les hommes et les femmes font la production et la commercialisation des chips de banane plantain au Cameroun. Cependant, ces activités sont exercées plus par les femmes. En effet, $88 \%$ des producteurs et $81 \%$ des commerçants sont des femmes. L'âge moyen des producteurs est de 34 ans et celui des commerçants de 26 ans.

Environ $65 \%$ des acteurs ont atteint le secondaire, $23 \%$ d'entre eux ont atteint le niveau primaire et $3 \%$ des acteurs n'ont pas été scolarisés. Seuls $10 \%$ des acteurs ont atteint le niveau supérieur.

Dans la production, le minimum d'années d'expérience est de moins d'un an et le maximum de 20 ans avec une moyenne de 5 ans. Quant à la commercialisation, le minimum d'ancienneté est de moins d'un an et le maximum de 35 ans avec une moyenne de 4 ans.

\section{Approvisionnement en banane plantain et en chips}

La banane plantain utilisée dans les unités de transformation provient essentiellement des différents marchés. Il faut cependant signaler qu'il existe dans les zones de production des marchés primaires de collecte qui alimentent les grands marchés 
dans les villes. Les cultivars de type bâtard (Bâtard) et faux corne (Abomienu, Red Ogoni, Orishele, Big Ebanga, etc.) sont plus sollicités par les producteurs à cause de leur grade (centième partie d'un quart de cercle) et des diamètres élevés. Ces derniers achètent la banane plantain sous forme de régime à des fréquences d'approvisionnement différentes. Les prix de vente des plantains sur les marchés varient fortement en fonction de l'offre et de la demande dans les marchés. Le prix moyen d'un régime d'environ $15 \mathrm{~kg}$ est de 2121 FCFA.

Les commerçants ont deux sources d'approvisionnement en chips. Environ 55\% d'entre eux s'approvisionnent au marché de vente en gros et $46 \%$ d'entre eux s'approvisionnent chez une revendeuse ou détaillante. Leurs fréquences d'approvisionnement varient en fonction de la demande de la clientèle.

\section{Processus de transformation de la banane plantain en chips}

La transformation de la banane plantain en chips se fait encore de manière artisanale et familiale. Elle est peu complexe et approximativement reste la même dans toute la zone d'étude. La Figure 1 montre les principales étapes de la transformation de la banane plantain en chips.

Les doigts de plantain sains sont lavés, épluchés et découpés. Le rendement de la pulpe par rapport au régime peut varier entre 45 à $60 \%$ de son poids en fonction des cultivars. Environ $71 \%$ des producteurs découpent les pulpes en lamelle et $29 \%$ d'entre eux les découpent en rondelle. Les deux formes de découpage n'ont pas toutes le même niveau d'appréciation auprès des acteurs. Il ressort de l'étude que $74 \%$ des acteurs préfèrent la forme lamelle alors que $26 \%$ de ceux-ci préfèrent la forme rondelle. La forme lamelle est plus appréciée des consommateurs parce qu'elle est plus croustillante, plus présentable et donne l'apparence d'être plus volumineuse. Quant à la forme rondelle, elle est appréciée des producteurs pour sa facilité de conservation. Certains producteurs ajoutent des arômes (sucre et/ou miel) aux pulpes découpées pour donner un goût particulier aux chips.

Les pulpes découpées sont ensuite introduites manuellement dans un bain d'huile (huile de palme extra raffinée, oléine ou stéarine) porté à la température de $160^{\circ}$ à 170 ${ }^{\circ} \mathrm{C}$. L'agitation des pulpes pendant la friture est indispensable pour éviter qu'elles ne se collent entre elles.

La phase d'égouttage suit la friture. Elle consiste à laisser les chips à température ambiante dans des récipients perforés pour faire partir l'excès d'huile au cours du refroidissement. Le conditionnement dans des sachets plastiques et leurs mises dans des récipients hermétiquement fermés constituent la dernière étape.

Le matériel couramment utilisé dans la transformation de la banane plantain en chips se compose d'une chipeuse manuelle (pour découper les pulpes), une friteuse, une écumoire (pour enlever les chips dans l'huile), des couteaux, des récipients perforés (pour extraire l'excès d'huile pendant l'égouttage).

Rentabilité de la production et de la commercialisation des chips de banane plantain

Rentabilité de la production des chips de banane plantain

La transformation de la banane plantain en chips a un coût. A travers les différents comptes d'exploitation mensuels moyens des différentes zones d'étude, nous dégagerons les différents coûts et marges (Tableau 1).

Il ressort de ce compte d'exploitation mensuel que les plus grandes dépenses liées aux charges variables se trouvent au niveau de l'achat du plantain, de l'huile et de la maind'œuvre. Notons que dans le processus de transformation, les acteurs font le recyclage de l'huile. La valeur mensuelle de l'amortissement de l'équipement pour la production des chips de banane plantain est faible, les coûts relatifs à l'équipement demandent des sommes modestes. 
L'analyse des comptes d'exploitation mensuels moyens montrent que la transformation de la banane en chips est une activité rentable dans les différentes villes. Le profit net obtenu est cependant variable selon les villes, avec un montant de 144676 FCFA à Boumnyebel, 122297 FCFA à Edéa, 114953 FCFA à Yaoundé et 97545 FCFA à Douala. L'activité est plus rentable dans les villes abritant des postes de péages routiers, car, dans ces villes il y a une forte demande du fait des voyageurs de l'axe Douala-Yaoundé.

\section{Rentabilité de la commercialisation des chips de banane plantain}

Comme la production, la commercialisation des chips de banane plantain a aussi un coût. A travers les différents comptes d'exploitation mensuels moyens des différentes zones d'étude, nous dégagerons les différents coûts et marges (Tableau 2).

La commercialisation des chips est une activité rentable et consiste juste à l'achat et la vente. Dans la plupart des cas, ces commerçants sont approvisionnés sur place par des transformateurs de plantains en chips. Cependant, il faut signaler que cette rentabilité est plus importante dans les postes de péages de Boumneybel et Edéa. Dans les autres villes, il est important de noter que ces commerçants ne vendent pas exclusivement les chips comme ceux des postes de péages. En effet, la vente des chips est une activité secondaire à l'activité principale qui est généralement «la vente des crédits de communications, communément appelé Call Box ». Sur une table ou kiosque de call-box, on peut $y$ rencontrer plusieurs autres friandises vendues à 100 FCFA comme les chips. Il s'agit entre autres des caramels, biscuits et autres produits de grignotage.

\section{Commercialisation des chips de banane plantain \\ Lieux de vente des chips de banane plantain}

Environ $82 \%$ des producteurs vendent les chips en gros aux revendeurs et $18 \%$ d'entre eux les vendent en détail et directement au consommateur final. Seules les villes de Douala et de Yaoundé regorgent les marchés de vente en gros de chips de banane plantain. A Douala, il existe un seul, il est situé à l'école publique de Déïdo. Il débute avant 5 heures du matin et se termine autour de 11 heures. A Yaoundé, il existe deux marchés de vente en gros situés à Mvog-Mbi et Anguissa. Ils se déroulent toute la journée. Le nombre de vendeurs et d'acheteurs qui fréquentent celui de Douala est largement supérieur à ceux de Yaoundé. La Photo 1 montre le marché de vente en gros de Douala situé à l'école publique de Deïdo.

Les observations directes dans la zone d'étude montrent que les chips de banane plantain sont revendues dans différents endroits répertoriés dans la Figure 2. Il ressort de cette figure que les chips de banane plantain sont plus revendues en bordure des routes (y compris les postes de péages) (80\%), devant les bureaux administratifs (9\%) et devant les agences de voyages $(5 \%)$. En plus de ces endroits spécifiques, les écoles, les lycées et les maisons sont dénombrées parmi les endroits où se revendent les chips. Il existe aussi des commerçants ambulants.

Dans les villes de Douala et Yaoundé, en dehors des commerçants ambulants qui vendent exclusivement les chips de banane plantain, la commercialisation de celles-ci est associée aux autres produits de grignotage (caramels, biscuits, cacahuètes diverses, etc.) vendus généralement à au plus 100 FCFA le sachet. Notons que la vente des chips de banane plantain et de ces autres produits accompagne généralement l'activité de «callbox» c'est-à-dire la vente des unités de crédit d'appels téléphoniques. Certains producteurs et commerçants, en fonction des lieux d'emplacement, payent des tickets de droit de place tous les jours à raison de 100 FCFA. Signalons aussi que les services communaux urbains sont assez vigilants pour remettre à l'ordre les commerçants qui s'installent anarchiquement dans les coins et les marchés 
de la ville. Au niveau des deux postes de péage, les commerçants concernés vendent exclusivement les chips de banane plantain. La Photo 2 montre des commerçants des chips de banane plantain au péage d'Edéa.

\section{Prix et unités de vente des chips de banane plantain}

Dans les différents points de vente de la zone d'étude, l'unité de vente des chips de banane plantain en gros ou détail est le sachet. Le poids du sachet varie de 31,9 à $67,1 \mathrm{~g}$. Le prix de vente unitaire en gros diffère d'un marché à un autre. Le prix de revente unitaire est de 100 FCFA dans toute la zone d'étude. Le Tableau 3 montre les différents prix de vente unitaire en gros d'un sachet de chip de banane plantain.

\section{Qualité des chips vendues}

La demande en chips dans les villes concernées par l'étude est forte. Presque toutes les couches socio professionnelles de la population sont des consommateurs de chips de plantain. Les chips sont vendues en rondelle ou en lamelle. Cependant, il faut tout de même reconnaître que certaines chips vendues ne sont pas toujours de bonne qualité à cause de certaines huiles de mauvaise qualité utilisée pour la friture. On obtient alors des chips non dorées et ayant une odeur désagréable. Lorsque le découpage est mal fait, les chips obtenues ont un excès d'huile et sont difficiles à conserver.

Du fait que la transformation des plantains en chips est encore au stade artisanale, dans certaines unités de production, les conditions hygiéniques sont très peu respectées et le produit obtenu a une qualité sanitaire douteuse et pas toujours appréciée des consommateurs de plus en plus exigeants. En effet, la production des chips se fait dans des lieux ouverts, laissant passer des quantités importantes de poussière qui se déposent sur les chips et l'équipement de production. En outre, au péage de Boumnyebel, la production des chips se fait à la périphérie des eaux stagnantes polluées et sujettes à des risques. Il serait important à cet effet de promouvoir le respect de normes hygiéniques lors de la production des chips.

\section{Circuits de commercialisation}

Le circuit de commercialisation des chips de plantain est peu complexe. Il est de deux types: court et direct. Le circuit direct est avantageux pour le producteur s'il écoule vite ses produits. Ce circuit permet également aux producteurs de retirer une plus grande valeur ajoutée des produits commercialisés. Le circuit court est avantageux à la fois pour le producteur et le commerçant. Le producteur écoule vite sa production et est épargné des contraintes liées à la commercialisation. Quant au commerçant, en plus du profit net qu'il peut avoir à la suite de la commercialisation, il est aussi épargné des contraintes liées à la production. Le circuit court permet d'atteindre le plus grand nombre de consommateurs comparativement au circuit direct.

\section{Difficultés rencontrées par les transformateurs et les commerçants de chips}

La transformation et la commercialisation des chips de banane plantain sont assurées par des unités artisanales et traditionnelles à l'état informel. Cette situation fait que les producteurs et commerçants de chips rencontrent plusieurs difficultés qui freinent le développement de la filière. Les Figures 3 et 4 présentent les difficultés évoquées par les enquêtés. Assurer la formation des producteurs et commerçants de chips sur les techniques modernes de transformation, de conditionnement et de commercialisation contribuerait fortement à lever les contraintes majeures auxquelles ils font face. 


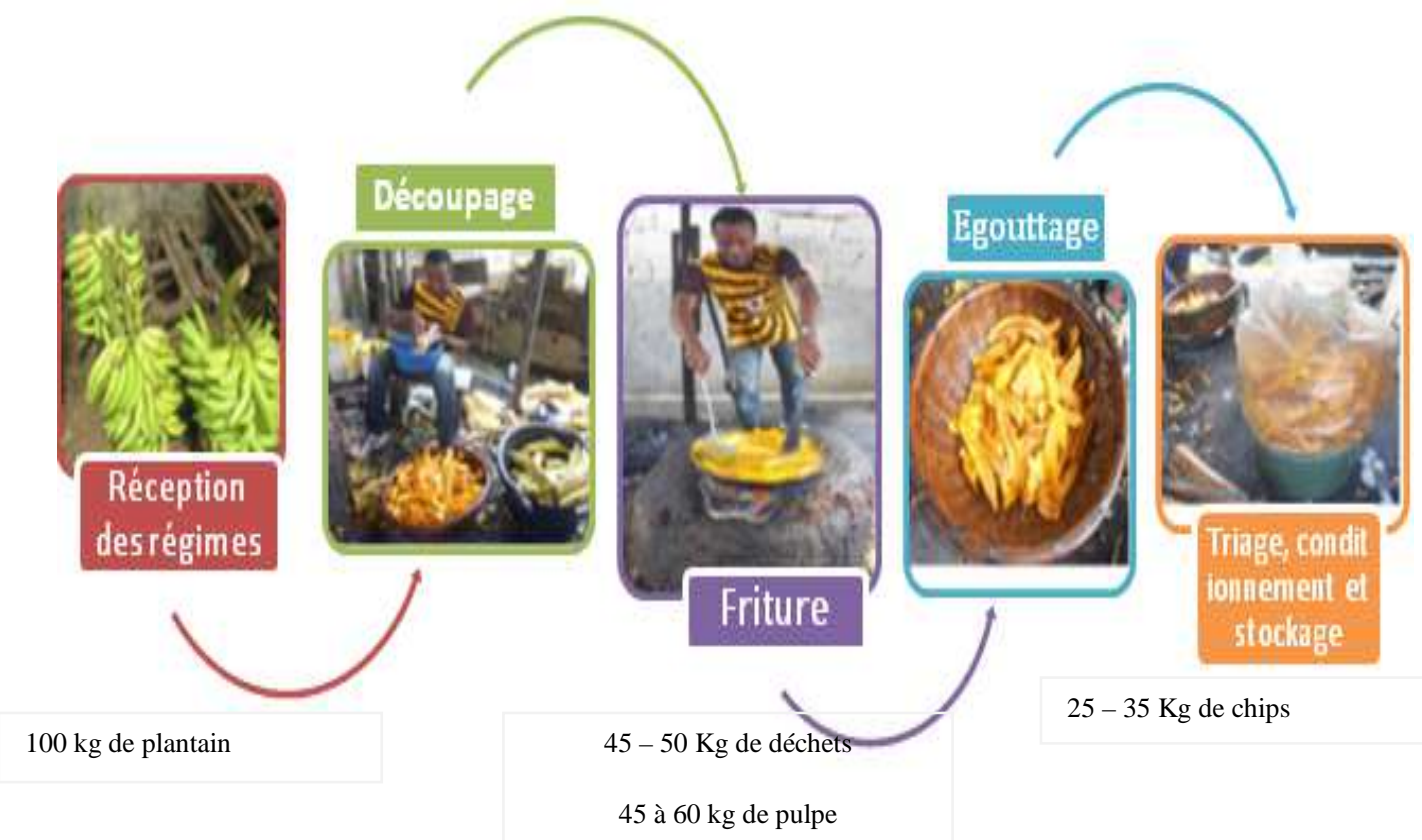

Figure 1: Processus de transformation de la banane plantain en chips.

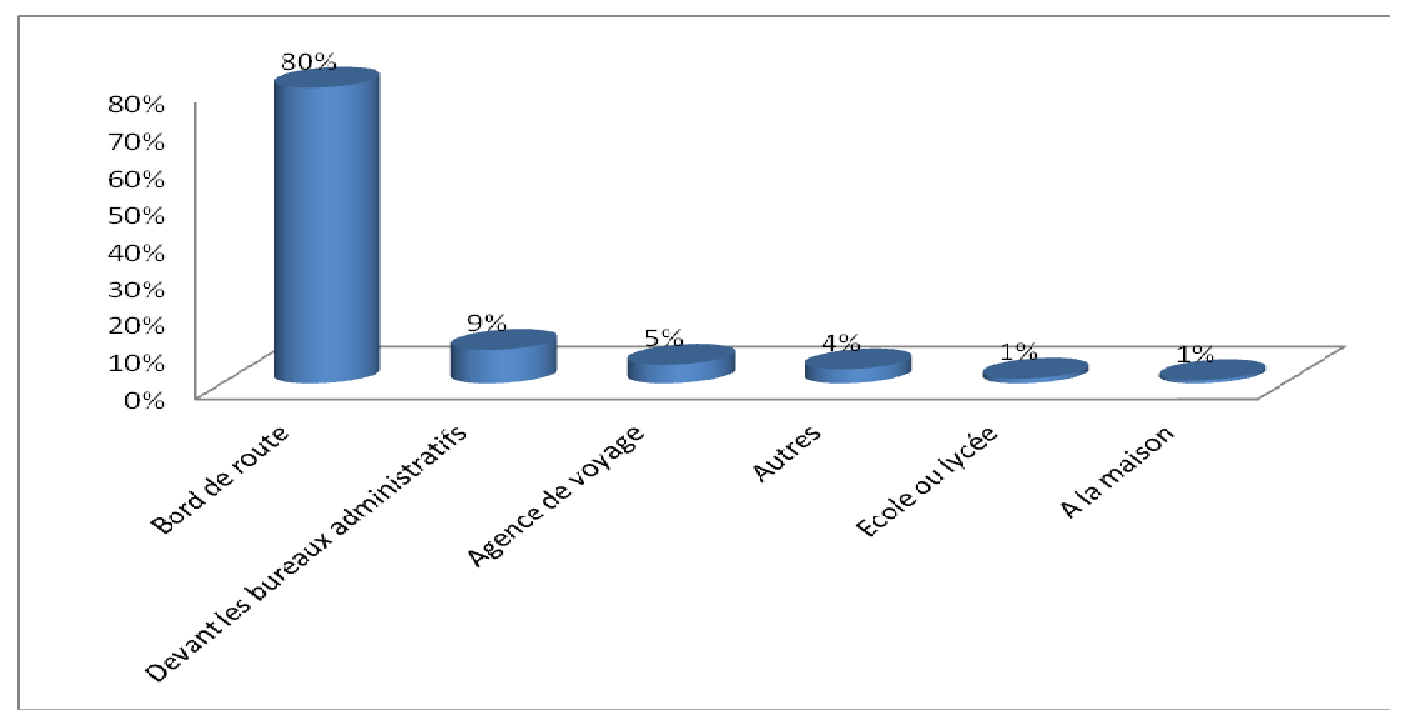

Figure 2: Lieux de vente des chips. 
G. H. FONGANG FOUEPE et al/Int. J. Biol. Chem. Sci. 10(3): 1184-1198, 2016

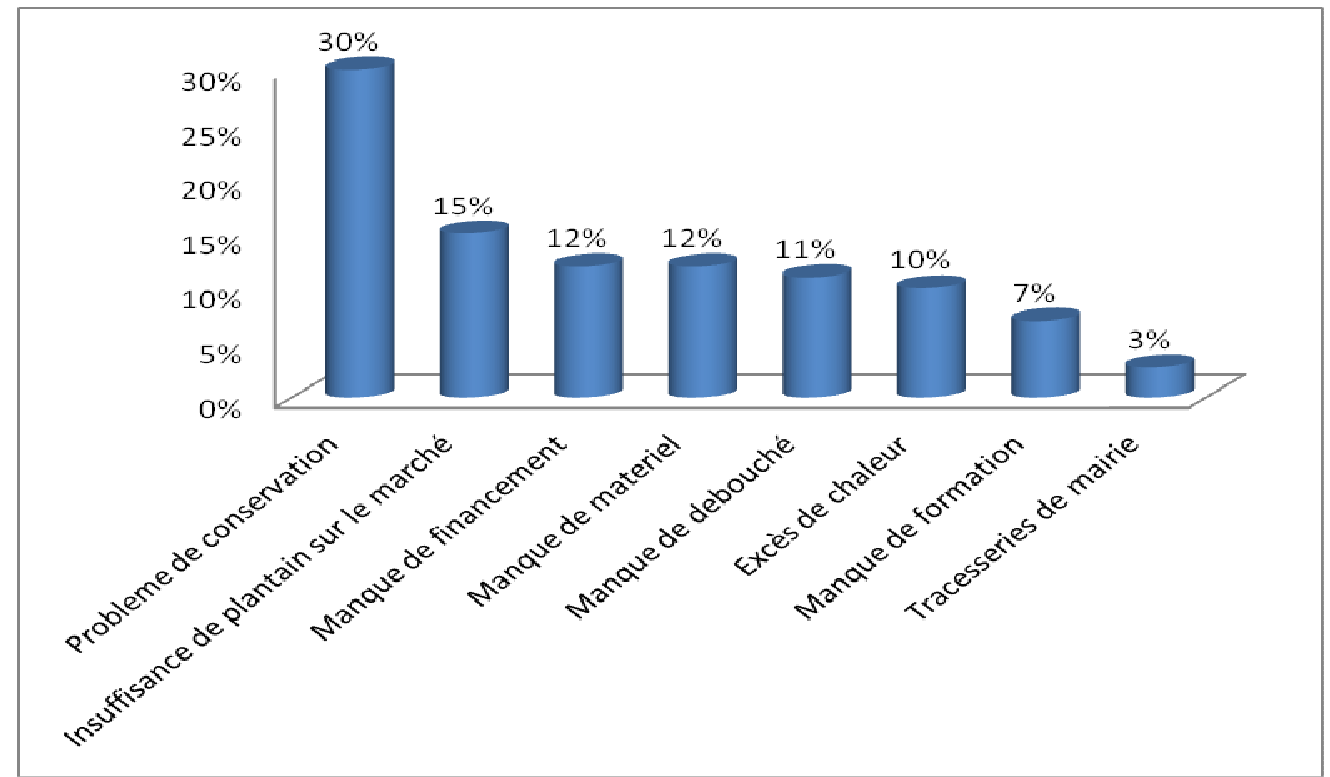

Figure 3 : Difficultés rencontrées par les producteurs de chips enquêtés.

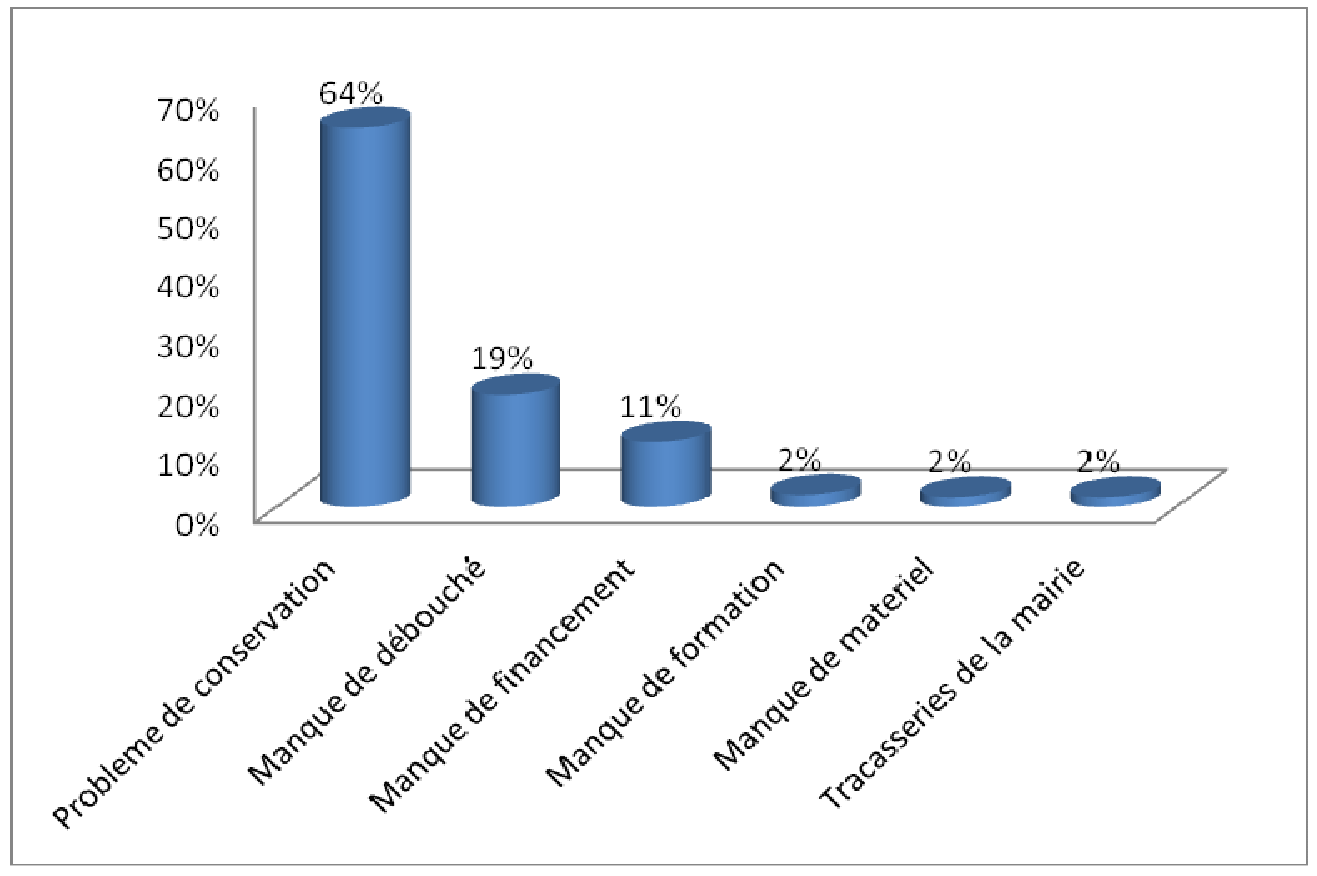

Figure 4 : Difficultés rencontrées par les vendeurs de chips enquêtés. 
G. H. FONGANG FOUEPE et al/Int. J. Biol. Chem. Sci. 10(3): 1184-1198, 2016

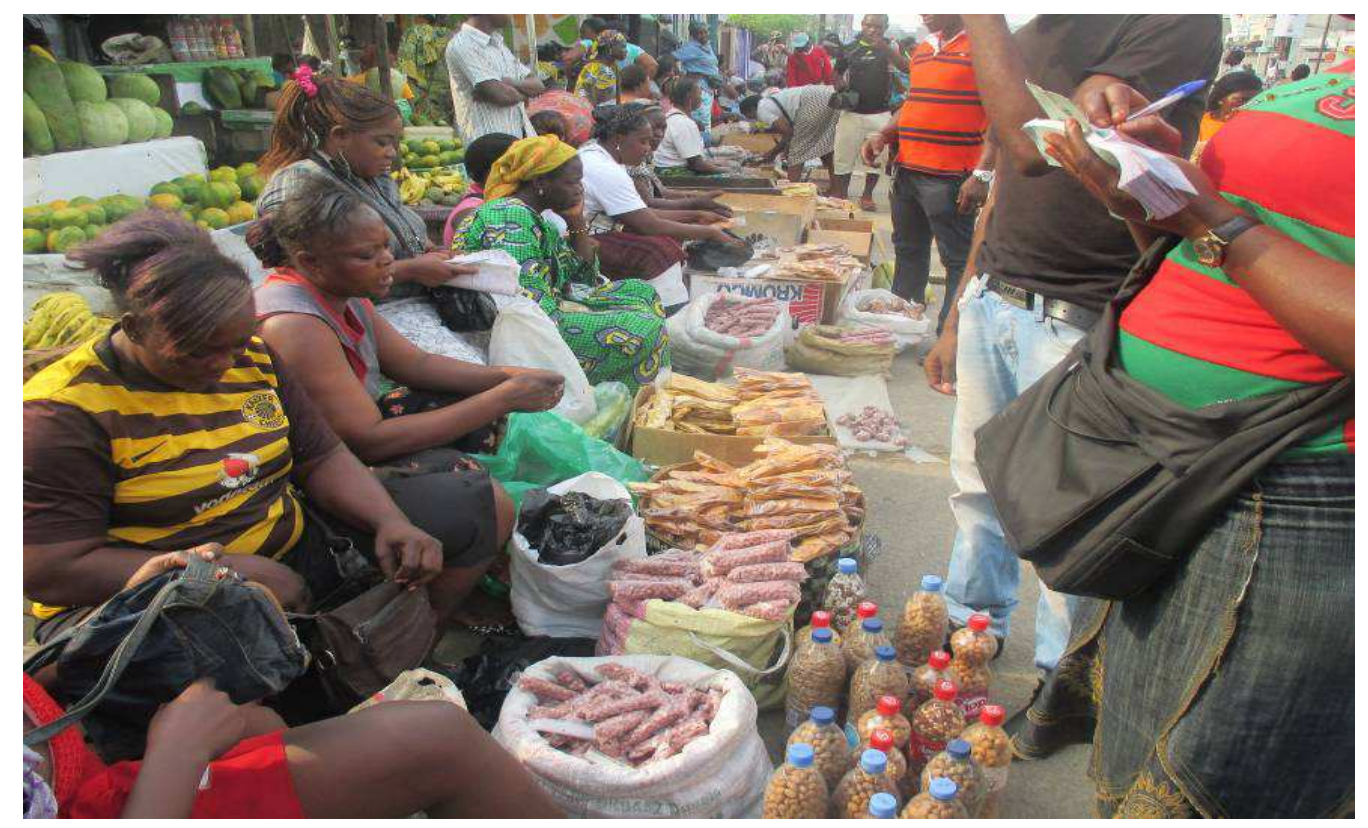

Photo 1 : Marché de vente en gros des chips à l'école publique de Deido à Douala.

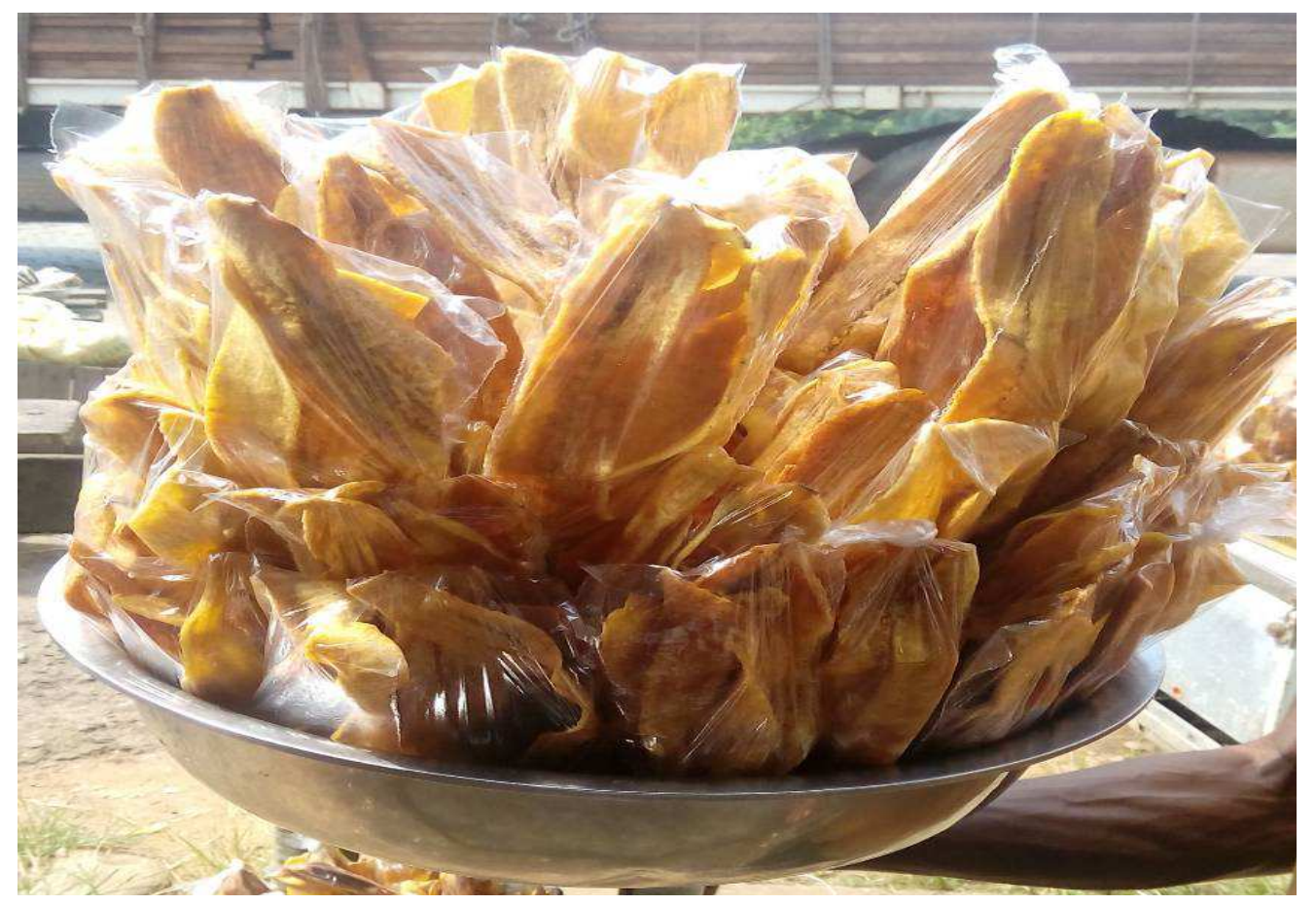

Photo 2 : Chips de banane plantain vendues au péage routier d'Edéa. 
G. H. FONGANG FOUEPE et al/Int. J. Biol. Chem. Sci. 10(3): 1184-1198, 2016

Tableau 1 : Compte d'exploitation mensuelle des transformateurs (production des chips).

\begin{tabular}{|c|c|c|c|c|c|c|c|c|c|c|c|c|c|}
\hline \multirow{3}{*}{$\begin{array}{l}\text { Villes } \\
\text { Rubrique }\end{array}$} & \multirow[b]{3}{*}{ Unités } & \multicolumn{3}{|c|}{ Boumnyebel } & \multicolumn{3}{|c|}{ Douala } & \multicolumn{3}{|c|}{ Edéa } & \multicolumn{3}{|c|}{ Yaoundé } \\
\hline & & \multicolumn{3}{|c|}{ FCFA } & \multicolumn{3}{|c|}{ FCFA } & \multicolumn{4}{|c|}{ FCFA } & \multicolumn{2}{|c|}{ FCFA } \\
\hline & & Quantité & $\mathbf{C U}$ & CT & Quantité & $\mathbf{C U}$ & CT & Quantité & $\mathbf{C U}$ & CT & Quantité & $\mathbf{C U}$ & CT \\
\hline \multicolumn{14}{|l|}{ Dépenses } \\
\hline Plantain & Régime & 96 & 1740 & 167040 & 44 & 2100 & 92400 & 48 & 1625 & 78000 & 112 & 3020 & 338184 \\
\hline Eau & Litre & 200 & 1 & 200 & 300 & 1 & 300 & 200 & 1 & 200 & 300 & 1 & 300 \\
\hline Bois & Tas & 120 & 100 & 12000 & 100 & 100 & 10000 & 338 & 100 & 23800 & 175 & 100 & 17500 \\
\hline Huile & Litre & 192 & 500 & 96000 & 110 & 500 & 55000 & 96 & 500 & 48000 & 200 & 500 & 100000 \\
\hline Main d'œuvre & $\mathrm{H} / \mathrm{J}$ & 16 & 2000 & 32000 & 24 & 2000 & 48000 & 48 & 1800 & 86400 & 48 & 1600 & 76800 \\
\hline Transport & forfait & 96 & 50 & 4800 & 44 & 100 & 4400 & 48 & 100 & 4800 & 112 & 100 & 11200 \\
\hline Emballage & Paquet & 12 & 1000 & 12000 & 4 & 1000 & 4000 & 19 & 1000 & 19000 & 42 & 1000 & 42000 \\
\hline Imprévus (10\%) & & & & 32404 & & & 21410 & & & 26020 & & & 58598 \\
\hline Total dépenses & & & & 356444 & & & 235510 & & & 286220 & & & 644582 \\
\hline \multicolumn{14}{|l|}{ Recette } \\
\hline Vente de chisp & Sachets & 6686 & 75 & 501450 & 4447 & 75 & 333525 & 5111 & 80 & 408880 & 9385 & 81 & 760185 \\
\hline Total recette & & & & 501450 & & & 333525 & & & 408880 & & & 760185 \\
\hline Profit brut & & & & 145006 & & & 98015 & & & 122660 & & & 115603 \\
\hline Amortissement & Valeur amortie & & & 330 & & & 470 & & & 363 & & & 650 \\
\hline Profit net & & & & 144676 & & & 97545 & & & 122297 & & & 114953 \\
\hline
\end{tabular}


G. H. FONGANG FOUEPE et al/Int. J. Biol. Chem. Sci. 10(3): 1184-1198, 2016

Tableau 2 : Compte d'exploitation mensuelle des commerçants.

\begin{tabular}{|c|c|c|c|c|c|c|c|c|c|c|c|c|c|}
\hline \multirow{3}{*}{$\begin{array}{l}\text { Villes } \\
\text { Rubrique }\end{array}$} & \multirow[b]{3}{*}{ Unités } & \multicolumn{3}{|c|}{ Boumnyebel } & \multicolumn{3}{|c|}{ Douala } & \multicolumn{3}{|c|}{ Edéa } & \multicolumn{3}{|c|}{ Yaoundé } \\
\hline & & \multicolumn{3}{|c|}{ FCFA } & \multicolumn{3}{|c|}{ FCFA } & \multicolumn{3}{|c|}{ FCFA } & \multicolumn{3}{|c|}{ FCFA } \\
\hline & & Quantité & $\mathbf{C U}$ & $\mathrm{CT}$ & Quantité & $\mathbf{C U}$ & CT & Quantité & $\mathbf{C U}$ & $\mathrm{CT}$ & Quantité & $\mathbf{C U}$ & CT \\
\hline \multicolumn{14}{|l|}{ Dépenses } \\
\hline Achats des chips & Sachets & 3528 & 75 & 264600 & 176 & 75 & 13200 & 1440 & 75 & 115200 & 480 & 81 & 38880 \\
\hline Transport & Forfait & & & 0 & & & 352 & & & 0 & & & 0 \\
\hline Total dépenses & & & & 264600 & & & 13552 & & & 115200 & & & 38880 \\
\hline Profit net & & & & 88200 & & & 4048 & & & 28800 & & & 9120 \\
\hline
\end{tabular}

Tableau 3: Prix de vente en gros d'un sachet de chip de banane plantain.

\begin{tabular}{lcccc}
\hline Ville & Prix moyen (FCFA) & Minimum (FCFA) & Maximum (FCFA) & N \\
\hline Boumnyebel & 75 & 75 & 75 & 20 \\
Douala & 76 & 71 & 80 & 186 \\
Edéa & 80 & 80 & 80 & 11 \\
Yaoundé & 81 & 75 & 90 & 110 \\
\hline
\end{tabular}




\section{DISCUSSION}

Cette étude porte sur l'analyse du système de transformation et de commercialisation des chips de banane plantain. Une analyse des caractéristiques socio économiques permet d'observer que l'activité de transformation et de commercialisation des chips est essentiellement féminine, les femmes étant fortement impliquées dans les activités de production $(88 \%)$ et de commercialisation (81\%). Ce pourcentage élevé pourrait s'expliquer par le fait que les femmes s'intéresseraient beaucoup plus qu'à la phase post récolte du plantain frais. C'est-à-dire qu'elles assurent d'une part, la distribution et la vente du plantain frais et d'autre part, la transformation du plantain frais. Elles s'intéressent aux cultures destinées à l'autoconsommation avec un cycle de production relativement court. Ngoma (2003) dans le même ordre d'idées note que $80 \%$ des producteurs dans la filière plantain d'approvisionnement de la ville de Douala sont les hommes car ce sont eux qui possèdent et gèrent la terre.

Ainsi, pour un développement de la sous filière transformation, un accent particulier doit être également mis sur les acteurs de sexe féminin étant donné que la production du plantain est assurée en majorité par les femmes dans de petites parcelles d'exploitation dans toutes les régions du Cameroun, en association avec d'autres cultures et de plus en plus dans des parcelles de culture pure. Ces actrices ont en général moins de 35 ans et ont suivi au moins des études primaires, voire secondaires. Elles sont le cour de la commercialisation locale et sous régionale de la banane plantain. Ceci est conforme à nos résultats, où la moyenne d'âge des commerçants est largement inférieure à celle des commerçants des villes de Douala et de Yaoundé. Ceci pourrait s'expliquer par le fait que la vente dans les voitures au péage est une activité physique exigeant assez d'énergie et impose d'être physiquement fort d'une part et d'autre part les enquêtes se sont faites en période de vacance pendant laquelle les élèves sont très présents au niveau des péages pour se faire un peu d'argent afin de préparer leur rentrée scolaire.

L'approvisionnement en bananes plantains s'effectue sur les marchés et auprès des producteurs connus avec qui a été établi un accord. La période d'octobre à mars est la période d'abondance de bananes plantains dans les zones de production. Il est souhaitable de disposer soi-même d'une plantation des variétés les plus aptes à la production des chips. Certaines variétés de plantain semblent plus adaptées et avantageuses pour la production des chips. Les producteurs ont donc des préférences quant à certaines variétés. Ces producteurs recherchent les gros doigts (Medou, Big ebanga, Agbaba) et les longs doigts (French clair). L'approvisionnement en huile de friture peut se faire auprès des huileries afin de bénéficier des prix préférentiels. Un contrat devrait être passé avec une huilerie pour la livraison de l'huile. La gestion de stocks des sachets et des cartons de conditionnement doit être suffisamment rigoureuse pour que les commandes soient passées à temps afin d'éviter des ruptures de stocks. Les pièces de rechange sensibles des équipements tels que la thermo-soudeuse, le robot-coupe ou la friteuse doivent être disponibles au magasin de l'unité de production.

Un autre fait marquant de cette étude, c'est que la transformation de la banane plantain en chips se fait encore de manière artisanale et familiale. La transformation permet aussi de réduire les pertes postrécoltes, accroître la durée de conservation, diversifier les formes d'utilisation et ajouter de la valeur au produit. Dans ces unités de transformations, les chips de banane plantain sont des produits issus de la friture dans un bain d'huile des rondelles de pulpe de fruit non mûr ou en début de maturation. 
L'humidité résiduelle et la teneur en matière grasse des chips de bonne qualité sont généralement inférieures à $5 \%$ et $35 \%$, respectivement (Newilah, 2005). Les chips salées sont les plus demandées et en fonction des exigences de la clientèle, des chips de plantain sucrées ou aromatisées peuvent être produites.

Comme l'activité reste dominée par les femmes, ces dernières manquent des moyens financiers pour la mise en place des unités modernes de transformation. Ce résultat est conforme à celui trouvé par Houngbo (2014) en Afrique de l'Ouest, dans les unités, les équipements de friture utilisés sont de qualités artisanales, fabriqués localement, en aluminium, avec des ustensiles en inox. Quelques rares unités utilisent des friteuses électriques ménagères. L'énergie électrique est rarement utilisée, le feu de bois étant préféré pour des raisons économiques.

Pour ce qui est de la rentabilité de l'activité, l'analyse des comptes d'exploitation de l'activité de production et de commercialisation des chips de plantain montre qu'elle est rentable. Elle procure ainsi des revenus substantiels à ces producteurs pour subvenir à leurs besoins quotidiens. En plus, la transformation à petite échelle des produits agricoles à haute valeur ajoutée peut conduire à la création d'emplois et à une sécurité alimentaire accrue. Une haute valeur ajoutée signifie que les transformateurs peuvent bénéficier d'un revenu raisonnable à partir d'échelles de production relativement petites (Axtell et Fellows, 2014).

Les chips de banane plantain sont plus revendues en bordure des routes (y compris les postes de péages) (80\%), devant les bureaux administratifs (9\%) et devant les agences de voyages (5\%). En plus de ces endroits spécifiques, les écoles, les lycées et les maisons sont dénombrées parmi les endroits où se revendent les chips. Il existe aussi des commerçants ambulants. Ce résultat concorde avec celui obtenu par Folefack et al.
(2008) qui stipulait que la plupart des produits agricoles transformés de manières artisanales et de consommation courante sont vendus dans tous les coins des villes.

Il faut aussi relever un fait non négligeable, celui de l'uniformisation des produits transformés. La qualité du produit est un élément important pour l'accessibilité et la compétitivité du produit sur le marché. Le problème de qualité des produits se pose à certains endroits. Ainsi, certaines chips vendues ne sont pas toujours de bonne qualité à cause de certaines huiles de mauvaise qualité utilisées pour la friture. Cette situation est récurrente sur les produits transformés en Afrique, ainsi plusieurs auteurs à l'instar de Diallo (1994) et Folefack et al. (2008) qui observaient les mêmes contraintes sur un produit transformé. Dans l'étude menée par Folefack en 2008, il observait aussi qu'à Maroua, la qualité du jus d'oseille de Guinée est loin d'être optimale, ce qui limite sa consommation et son accessibilité sur le marché formel. En effet, tout cela part du fait que le mode de transfert de la connaissance sur la technique de transformation des produits est informel et traditionnel, et cette activité est effectuée à l'échelle artisanale dans les ménages, avec des conditions de transformation, de conservation et de commercialisation peu adéquates et peu adaptées.

Même lorsque les débouchés existent, il y a fréquemment de gros obstacles au développement de la filière agro-alimentaire. Les acteurs de la transformation et la commercialisation des chips de banane plantain rencontrent plusieurs difficultés qui freinent le développement de la filière. Cependant, il faut signaler que la contrainte majeure reste celle de la conservation des produits transformés. En effet, les chips sont des produits fragiles et susceptibles de se casser et de perdre leur clarté en cas d'une mauvaise conservation. Les chips endommagées deviennent très difficiles à 
écouler. Les innovations technologiques visant à améliorer la durée de conservation, les aptitudes au stockage et l'attrait pour le consommateur doivent s'accompagner d'une bonne évaluation des améliorations qui s'imposent.

\section{Conclusion}

Cet article a permis de ressortir les principales caractéristiques socioéconomiques des producteurs et commerçants de chips au Cameroun. L'activité de production et de commercialisation des chips de banane plantain reste en majorité une activité des femmes. Les unités de production des chips de banane plantain sont artisanales et familiales. Le procédé de production des chips de banane plantain est peu complexe et approximativement le même dans toutes les zones d'étude. L'activité de production et de commercialisation des chips de banane plantain est rentable et procure des revenus substantiels aux ménages pauvres en milieu urbain et rural. Bien que l'activité soit florissante, les conditions de production des chips sont peu hygiéniques et peuvent causer des dommages à la santé du consommateur. Une formation des producteurs et commerçants de chips sur les techniques modernes de transformation, de conditionnement et de commercialisation est nécessaire et permettrait d'améliorer significativement les performances de cette filière.

\section{CONFLIT D'INTERET}

Les auteurs déclarent qu'il $\mathrm{n}$ y a pas de conflit d'intérêt.

\section{CONTRIBUTIONS DES AUTEURS}

GHFF et DPF sont des investigateurs principaux de cet article. Ils ont contribué à la conception méthodologie, la collecte, l'analyse des données et rédaction du manuscrit ; ZPP a contribué à la collecte des enquêtes sur le terrain et l'analyse statistique ;
$\mathrm{AB}$ et $\mathrm{PN}$ ont contribué à la correction du manuscrit.

\section{REFERENCES}

Bikoï A, Nkapnang D, Ndemba B, Ziki E, Keleke S, Ebongue JP. 2012. Analyse des circuits de commercialisation du plantain dans les plateformes transfrontalières Ntem, Oubangui et Congo. Planta Innovation n91-94, juillet 2011-juin 2012, CARBAP.

Diallo A. 1994. La transformation des produits agricoles locaux au Burkina Faso: des innovations en échec. Analyse de cas sur la méconnaissance du marché. Orstom, Ouagadougou, Février 1994.

DSDSR. 2005. Document de stratégie du développement du secteur rural, www.minepat.gov.cm/index.php?option =com_docman\&task. Consulté le $15 / 05 / 15$.

DSCE. 2009. Document de Stratégie de la Croissance et de l'Emploi, Cameroun. http://www.afdb.org/fileadmin/uploads/a fdb/Documents/Project-and

Operations/Cameroon\%20DSCE2009.pd f. Consulté le 10/06/15.

Efandem C, Kwa M, Temple L, David O. 2005. Production de plantain dans la zone péri urbaine de Yaoundé : Identification des contraintes et incidences sur l'origine et la quantification des flux des marchés de la ville, en Agriculture et développement urbain en Afrique de l'Ouest et du Centre, Atelier, Yaoundé, Cameroun, 31 octobre-03 Novembre 2005, CIRAD, Yaoundé, Cameroun.

Fellows PJ, Axtell B. 2014. Opportunities in Food Processing: A Handbook for Setting up and Running a Smallscale Business Producing High-Value Foods. ACP-EU Technical Centre for Agricultural and Rural Cooperation (CTA): Wageningen. 
Folefack DP, Njomaha C, Djouldé DR. 2008. Diagnostic du système de production et de commercialisation du jus d'oseille de Guinée dans la ville de Maroua. Tropicultura, 26(4) : 211-215.

Houngbo E. 2014. Etude régionale sur la commercialisation de la banane plantain en Afrique de l'Ouest. CORAF/ WECARD, Rapport final.

Moustier P, Pages J. 2002. Le périurbain en AFRIQUE : Une agriculture en marge ?

Le Courrier de l'Environnement, 32 (Décembre 2002).

Newilah NG, 2005. Utilisation alimentaire, caractérisation physico-chimique et Biochimique des fruits de quelques cultivars et hybrides de bananiers et plantains produits au Cameroun. Thèse de Doctorat 3ème cycle de Biochimie. Département de Biochimie, Université de Yaoundé I. Cameroun, 131p.

Nkendah R, Ako E, Tamokwe B, Nzouessin C, Njoupouognigni $M$, Melingui E, Azeufouet A. 2011. Cameroun. Le commerce transfrontalier informel des produits agricoles et horticoles. Économie Rurale, 324, juillet-août 2011, mis en ligne le 30 juillet 2013, consulté le 09 mai 2016. URL :

http://economierurale.revues.org/3083.

Nkendah R, Nzouessin CB, Temple L. 2007. Economic analysis of the spatial integration of plantain markets in Cameroon. African Journal of Economic Policy, 14(1): 57-82.
Nkendah R, Temple L. 2003. Pression démographique et efficacité technique des producteurs de banane plantain de l'Ouest Cameroun. Cahiers d'Agricultures, 125 (5): 333-339.

Ngoma MMB. 2003. Distribution des revenus au sein de la filière plantain d'approvisionnement de la ville de Douala. Mémoire de fin d'études. FASA. Université de Dschang. Cameroun. 78p.

Planta Innovation. 2011. Bulletin de liaison des plateformes d'Innovation sur les filières bananes et plantains en Afrique ; $\mathrm{N}^{\circ}$ 5-6; Janvier -Juin 2011. 19p.

Tchango Tchango J, Ngalani JA, Newilah G, Emaga T. 2009. Les chips de banane et de plantain: production artisanale. CARBAP, Cameroun.

Temple L, Lançon F, Palpacuer F, Paché G. 2011. Actualisation du concept de filière dans l'agriculture et l'agroalimentaire. Economies et Sociétés, Série «Systèmes agroalimentaires» $\mathrm{AG} \mathrm{n}^{\circ} \mathbf{3 3}, 7 \mathrm{p}$.

Wandji AA. 2015. Dynamique des marchés transfrontaliers de la banane plantain de la vallée du Ntem. Mémoire de fin d'études, ESSEC Douala/CARBAP, Université de Douala, 103 p. 